\title{
EDITORIAL
}

\section{CORTISONE IN SYPHILIS OF THE EYE}

The therapeutic successes scored by arsphenamine, bismuth compounds, and penicillin against syphilis in general have been offset by a relative failure in the management of two late complications which affect the eye. These two conditions - syphilitic optic atrophy and interstitial keratitisare still too common and still cause much individual suffering and disability as a result of impaired vision. Such blindness, sometimes only partial but too often total, overtakes the individual at an active time of life when family and economic responsibility may be maximal. In their acute stage, the management of these two eye complications has presented unusual difficulty, as often they respond satisfactorily neither to routine antisyphilitic treatment nor to the additional use of pyretotherapy. The prognosis in syphilitic optic atrophy is poor, and, except in the earliest stage, the condition frequently progresses to total blindness in spite of the most active treatment. Again, the outcome of interstitial keratitis defies accurate forecast in the individual patient. It may develop in the congenital case which has previously escaped detection and treatment, but not uncommonly it attacks the patient who has received what is regarded as adequate anti-syphilitic treatment and may actually appear during the course of active therapy. One or both eyes may be affected and relapse is far from infrequent. The pathological course of each individual attack is extremely variable, some cases clearing rapidly in a week or two, whilst others smoulder on for many months in spite of expert and comprehensive treatment. The degree of residual corneal scarring is equally variable. Interstitial keratitis is a late manifestation of congenital syphilis; the pathological process is not exactly understood, but there are grounds for believing that it results from an allergic hypersensitivity of the tissues. This theory is still widely held in spite of the failure of the antihistamine drugs to benefit the condition. In contrast, syphilitic iritis, which is usually a complication of the secondary stage, responds well to routine treatment and relapse is not seen provided therapy is adequate.

This, then, was the unsatisfactory position in 1949 when Hench, treating cases of rheumatoid arthritis who also had iritis, noted the dramatic effect of cortisone on the inflammatory process in the eyes. Research and clinical trials have proceeded rapidly in the past 3 years and although far from complete, a working knowledge of how to use cortisone and what may be expected of it is now emerging. Dr. G. O. Horne, addressing the Medical Society for the Study of Venereal Diseases (January 25, 1952), indicated the possible applications of ACTH and cortisone in syphilis, and on November 28, 1952, the Society heard Dr. A. N. Ashworth describe his considerable experience of the effects of cortisone in syphilitic eye disease. This address appears in the present issue of the Journal together with the discussion which the paper stimulated.

The adrenal cortex elaborates a number of steroid hormones of which cortisone (17-hydroxy-11dehydrocorticosterone or Compound $\mathrm{E}$ ) is the most potent. The effects of these hormones fall into three main categories (Woods, 1952) :

(1) hormones with an electrolyte-regulating effect which cause retention of sodium chloride, increased excretion of potassium, and increased volume of plasma and extracellular fluid ;

(2) hormones which modify carbohydrate, fat, and protein utilization, cause lysis of fixed lymphoid tissue, and depress the level of circulating eosinophils ;

(3) hormones related to testosterone which produce androgenic effects, e.g. hirsutism.

There is some overlap between these three categories, but the effects of cortisone essentially fall into the second. Administration of ACTH (pituitary adrenocorticotrophic hormone) stimulates the adrenal cortex to produce these steroid hormones in unusual amount and, embracing all three fields of action, causes more widespread effects than those of cortisone.

The prolonged, systemic use of either ACTH or cortisone can cause certain serious physiological disturbances, including retention of salt and water with a resulting hypertension, relatively insulinresistant hyperglycaemia, and Cushing's syndrome with the characteristic facies, hirsutism, and amenorrhoea. As a consequence, this hormone therapy should be restricted to patients in hospital where 
the laboratory facilities necessary for the control of treatment are available: weight and blood pressure recordings and tests for glycosuria should be performed daily ; the fasting blood sugar should be estimated before treatment and subsequently at intervals of 4 to 6 days ; daily eosinophil counts should be made to indicate the general physiological response and to guide the daily dosage of the hormone. To guard against potassium loss, $3 \mathrm{~g}$. potassium chloride daily is recommended if therapy is prolonged.

While ACTH must be given parenterally as it acts only by stimulating the adrenal cortex, cortisone is effective locally in the anterior segment of the eye ; it can be administered in ointment or drops, or by subconjunctival injection. The last method was favoured at first but it is now agreed that equally good results follow the use of drops by day and ointment by night to avoid disturbing sleep. Local application enjoys many advantages of which the chief is freedom from systemic sideeffects, and local treatment may thus be maintained for many months without fear of complications ; it is also economical in use and makes possible ambulant treatment. Cortisone applied as ointment and drops enables treatment to be "longer, safer, cheaper, and ambulant" (Duke-Elder, 1951). Unfortunately, these advantages of local treatment can only be applied to disease of the anterior segment of the eye ; when the posterior segment and optic nerve are involved, the hormone must be administered parenterally.

It is clear that cortisone has no curative powers per se, but it exerts a potent effect in controlling the inflammatory and exudative responses to infection and allergic reactions. It influences not the cause of the disease but the reaction of the tissues, and this suppressive effect simulates cure often with dramatic rapidity. However, unless the underlying cause is self-limiting or susceptible to specific treatment, the condition will recur if cortisone is withdrawn before the causal factor ceases to operate. Cortisone is thus only an adjuvant to the complete treatment of syphilitic interstitial keratitis, but a majority of those with most experience of its use believe that by producing rapid resolution of the inflammation and exudation, it spares the patient much physical discomfort and probably also saves his vision by eliminating or greatly reducing residual scarring of the cornea. For these reasons, cortisone must be started without delay and diagnosis must certainly not await serological confirmation. The diagnosis of late congenital syphilis can often be made with confidence on clinical grounds alone, and it should be remembered that at least 15 per cent. of such cases will be sero-negative (Laird, 1950). Close cooperation between ophthalmologist and venereologist has always been fruitful where it has existed in the past; the advent of cortisone and the need for its early exhibition in the management of interstitial keratitis makes this cooperation imperative.

\section{REFERENCES}

Duke-Elder, S. (1951). Brit. J. Ophthal., 35, 637.

Horne, G. O. (1952). British Journal of Venereal Diseases, 28, 106. Laird, S. M. (1950). Ibid., 26, 143.

Woods, A. C. (1952). Brit. J. Ophthal., 36, 401. 